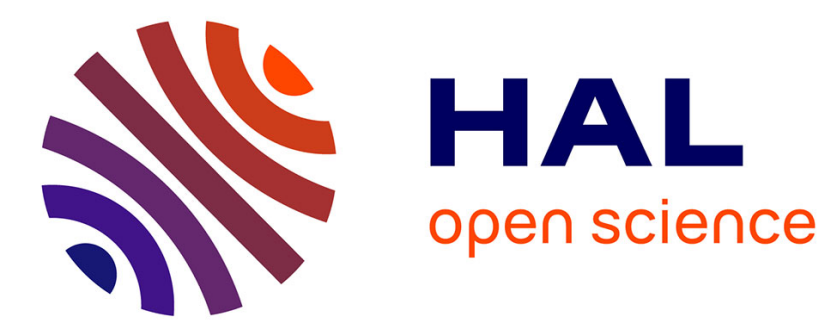

\title{
Cinétique de dissolution d'un dépôt $\mathrm{Fe} / \mathrm{Cu}$
}

\author{
S. Delage, B. Legrand, F. Soisson, T. Bigault, A. Saúl, G. Tréglia
}

\section{To cite this version:}

S. Delage, B. Legrand, F. Soisson, T. Bigault, A. Saúl, et al.. Cinétique de dissolution d'un dépôt Fe/Cu. Journal de Physique IV Proceedings, 1996, 06 (C7), pp.C7-151-C7-154. 10.1051/jp4:1996717 . jpa-00254505

\section{HAL Id: jpa-00254505 https://hal.science/jpa-00254505}

Submitted on 1 Jan 1996

HAL is a multi-disciplinary open access archive for the deposit and dissemination of scientific research documents, whether they are published or not. The documents may come from teaching and research institutions in France or abroad, or from public or private research centers.
L'archive ouverte pluridisciplinaire HAL, est destinée au dépôt et à la diffusion de documents scientifiques de niveau recherche, publiés ou non, émanant des établissements d'enseignement et de recherche français ou étrangers, des laboratoires publics ou privés. 


\title{
Cinétique de dissolution d'un dépôt $\mathrm{Fe} / \mathrm{Cu}$
}

\author{
S. Delage, B. Legrand, F. Soisson, T. Bigault*, A. Saúl* et G. Tréglia* \\ SRMP/DECM, CEA Saclay, 91191 Gif-sur-Yvette cedex, France \\ * CRMC2-CNRS, Campus de Luminy, Case 913, 13288 Marseille cedex 9, France
}

\begin{abstract}
Résumé: La prise en compte des moteurs de ségrégation superficielle dans un modèle cinétique (KTBIM) permet de rendre compte des cinétiques de dépôt-dissolution observées expérimentalement. Nous nous proposons de mettre en évidence le comportement des premiers plans près de la surface dans le cas d'un dépôt de fer sur un substrat de cuivre: une (ou deux) couche(s) flottante(s) de cuivre apparaissent sur le dépôt de fer avant la dissolution de celuici, en très bon accord avec les observations expérimentales. Le concept d'équilibre local nous permet de faire le lien entre les profils de concentration observés lors de la cinétique de dissolution et les profils d'équilibre de la couche mince dite équivalente.
\end{abstract}

\section{INTRODUCTION}

Des études expérimentales de dépôts de $\mathrm{Fe}$ sur $\mathrm{Cu}$ ont révélé l'existence de phénomènes remarquables au voisinage de la surface: il apparaît sur des temps très courts une ou plusieurs couches "flottantes" de cuivre recouvrant le dépôt de fer [1]. Nous nous proposons ici de modéliser ce comportement et d'en expliquer l'origine dans le cas de dépôts minces. Ce travail constitue la première étape vers une meilleure compréhension des cinétiques de dissolution de dépôts épais $\mathrm{Fe} / \mathrm{Cu}$ et $\mathrm{Cu} / \mathrm{Fe}$, ainsi que de leur liaison avec les cinétiques de ségrégation superficielle dans les alliages $\mathrm{Fe}(\mathrm{Cu})$ à haut intérêt technologique dans l'industrie nucléaire. La comparaison de ces différentes cinétiques est cependant rendue compliquée par la variété des structures cristallographiques, le fer étant Cubique Centré (C.C.) dans la gamme de température considérée et le cuivre ayant la structure Cubique à Faces Centrées (C.F.C.). Pour s'affranchir de cette difficulté, tous les calculs seront faits dans la structure C.C, même si ce choix n'est pas optimum pour le traitement des dépôts $\mathrm{Fe} / \mathrm{Cu}$ présenté ici. Nous reviendrons sur l'influence de la structure dans la conclusion.

La nécessité de prendre en compte les moteurs de la ségrégation superficielle pour décrire les cinétiques de dissolution a été récemment montrée [2]. Nous avons ainsi étudié les cinétiques de dépôt-dissolution de $\mathrm{Fe}$ sur $\mathrm{Cu}$ à l'aide d'un modèle cinétique (KTBIM : Kinetic Tight Binding Ising Model) s'appuyant sur les trois moteurs de la ségrégation de surface: l'effet de tension superficielle, l'effet d'alliage et l'effet de taille [3].

Le système $\mathrm{Fe}-\mathrm{Cu}$, qui a une forte tendance à la démixtion en volume, présente à l'équilibre une ségrégation superficielle de cuivre importante due à un effet de tension superficielle (la différence des énergies de surface pour la face compacte ${ }^{l}$ est estimée à $272 \mathrm{meV} / a t$ [5]). L'effet d'alliage, responsable des transitions de phase en surface [6], est estimé à partir des limites de solubilité en volume [7]. Ceci conduit à des interactions effectives d'alliage [8] entre premiers voisins de $-36 \mathrm{meV}$, l'interaction entre seconds voisins étant estimée égale à la moitié de celle entre premiers voisins pour la structure cubique centrée selon les calculs de structure électronique [8]. L'effet de taille [9] quant à lui est négligeable dans le cas du système $\mathrm{Fe}-\mathrm{Cu}$.

Nous avons effectué les calculs à $\mathrm{T}=700 \mathrm{~K}$, température pour laquelle le coefficient de diffusion est égal à 2 $10^{-19} \mathrm{~cm}^{2} / \mathrm{s}$ [7]. Les cinétiques seront reportées en fonction de $\mathrm{t} / \mathrm{t}_{0}$, où to est le rapport $\frac{a^{2}}{D}$ (a: paramètre de maille). Dans une large gamme de température, une variation de $\mathrm{T}$ se traduit essentiellement par une homothétie sur les temps due à la variation de $\mathrm{D}$ et donc de to.

Nous avons tout d'abord étudié la dissolution d'une puis de deux monocouches (MC) de fer déposées sur un substrat de cuivre. Nous relions ensuite les comportements observés durant la cinétique à la ségrégation d'équilibre via le concept d'équilibre local. 


\section{RÉSULTATS}

\subsection{Dépôt d'une monocouche de fer sur un substrat de cuivre}

Considérons un dépôt d'une monocouche de fer sur un substrat de cuivre (étape A1, figure 1a). Dans un premier temps, l'effet de tension superficielle induit un enrichissement quasi-instantané de la surface $(\mathrm{p}=0)$ en cuivre tandis que le dépôt de fer se répartit sur les trois plans sous-jacents $(\mathrm{p}=1,2,3)$. Le plan $p=1$ subit alors aussi une remontée du cuivre, et l'on obtient deux couches de cuivre $\left[\mathrm{c}_{0}(\mathrm{Fe})=0\right.$, $\left.c_{1(F e)}=5 \cdot 10^{-2}\right)$ ] "flottant" sur deux plans $\mathrm{p}=2,3$ de compositions variables $\mathrm{Fe}_{\mathrm{x}(\mathrm{t})} \mathrm{Cu}_{1-\mathrm{x}(\mathrm{t})}$; cet état perdure sur des temps relativement longs avec une dérive lente de la composition des couches 2 et 3 (étape A2).

Enfin, pour des temps plus longs (étape A3), on observe une dissolution classique de type fickien sur tous les plans situés sous la surface (la surface conservant une concentration nulle de fer). L'évolution temporelle de la concentration en fer des sept premiers plans de surface est donnée figure $1 \mathrm{~b}$ en fonction de $\sqrt{t / t_{0}}$. Nous verrons au paragraphe 3 comment interpréter l'étape lente de la cinétique, correspondant au stade A2, ainsi que sa disparition (pour laisser place à l'étape A3) en terme d'équilibre local [10].

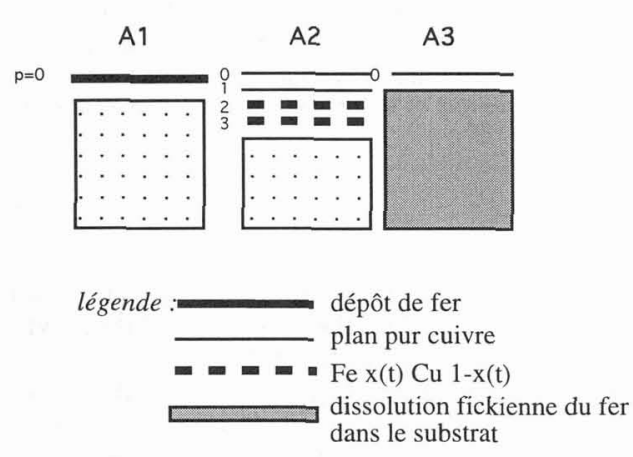

figure la

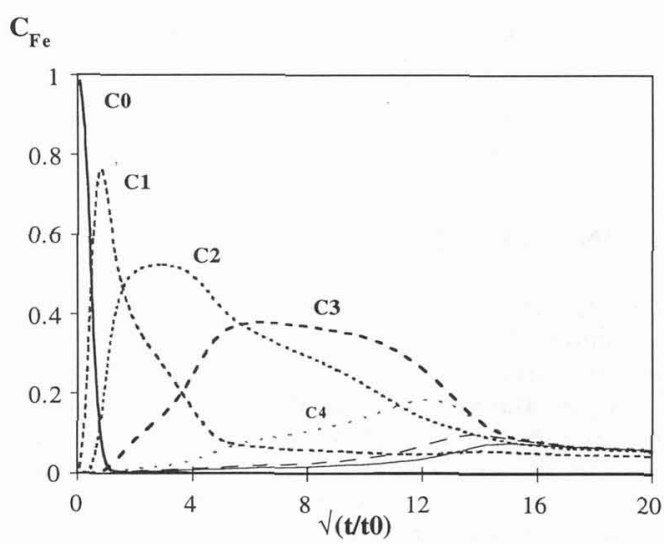

figure $1 b$

Figure 1: Cinétique de dissolution d'une couche de fer déposée sur un substrat de cuivre. a) évolution schématique; b) résultat du modèle KTBIM (les indices des concentrations correspondent à la numérotation des plans de la figure 1a)

\subsection{Dépôt de deux monocouches de fer sur un substrat de cuivre}

Considérons maintenant un dépôt de deux monocouches de fer sur un substrat de cuivre (étape B1, figure 2a).

Comme dans le cas précédent, le plan de surface s'enrichit en cuivre en des temps très courts. Cependant on observe ici la persistance d'une monocouche flottante de cuivre, les plans sous-jacents $(\mathrm{p}=1,2)$ étant très riches en fer (étape B2, figure $2 b$ ). Durant cette étape d'évolution lente, la quantité de fer contenue dans les plans 1,2 et 3 passe pratiquement de $2 \mathrm{MC}$ à $1 \mathrm{MC}$. On assiste alors à une variation très rapide des concentrations des premiers plans sous la surface, le "précipité" de fer migrant soudainement des plans 1 et 2 vers les plans 2 et 3 tout en se dissolvant lentement (figure 2c). Remarquons que cette étape B3, dans laquelle le précipité de Fe contient l'équivalent de moins d'une monocouche de fer, est très similaire à l'étape A2 obtenue précédemment dans le cas du dépôt d'une monocouche. La fin de la dissolution est également très comparable à celle de la figure $1 \mathrm{~b}$, les gradients de concentration devenant très faibles sauf pour le plan 0 et le plan 1 dans une moindre mesure.

Nous nous proposons de montrer comment la notion d'équilibre local entre les premiers plans de surface permet d'expliquer les étapes B2 et B3, l'explication pouvant être transposée pour le cas d'une monocouche de fer déposée sur du cuivre. 

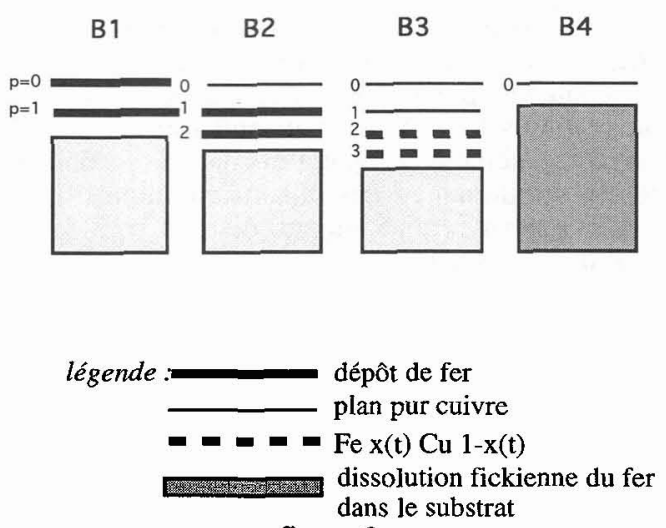

figure $2 \mathrm{a}$

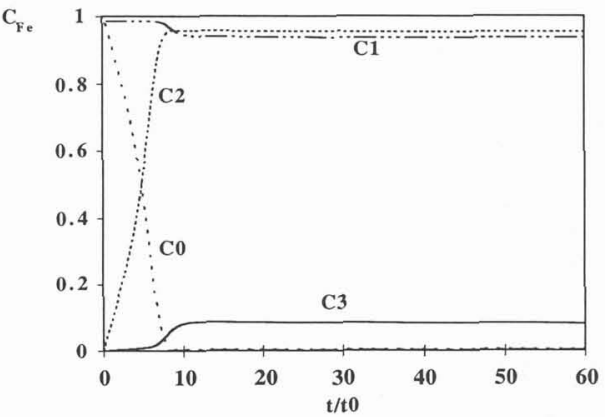

figure $2 b$

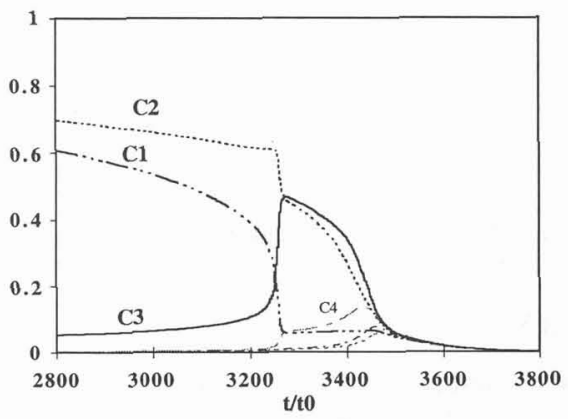

figure $2 \mathrm{c}$

Figure 2: Cinétique de dissolution de deux monocouches de fer déposées sur un substrat de cuivre. a) évolution schématique; b),c) résultat du modèle KTBIM

\subsection{Equilibre local}

Selon Laguës et Domange [10], on peut supposer que les plans situés au voisinage de la surface sont en équilibre 'local' au bout d'un temps négligeable devant le temps moyen de diffusion thermique des couches déposées. Nous nous proposons ici de montrer que les stades B2 et B3 correspondent à des équilibres locaux, directement reliés à des profils de ségrégation d'équilibre.

Lors des stades B2 et B3, le potentiel chimique par plan [11] se révèle être constant sur les cinq premiers plans de surface. Ceci signifie que les flux de matière entre ces plans se compensent et indique l'établissement de l'équilibre local sur cette épaisseur.

Pour mettre en évidence cet équilibre local à un instant $\tau_{0}$, introduisons le concept de couche mince équivalente $(\mathrm{CME})$, dans le cas présent une couche mince de cinq plans, de concentration moyenne identique à la concentration moyenne des cinq premiers plans à l'instant $\tau_{0}$ de la cinétique. Fixons de plus la concentration du cinquième plan à sa valeur à l'instant $\tau_{0}$.

Nous nous proposons de vérifier que l'état d'équilibre de la $\mathrm{CME}$ est très proche du profil cinétique à $\tau_{0}$. La figure 3, sur laquelle sont portés les profils de concentration cinétiques et d'équilibre en fonction de la concentration moyenne des cinq premiers plans, montre la validité de cette notion d'équilibre local. Celle-ci permet d'avancer dans la compréhension des particularités des cinétiques. Ainsi la variation brutale des concentrations entre les stades B2 et B3 correspond à la fin de la stabilité d'un équilibre où le précipité se trouve sur les plans 1 et 2 . Lors de la cinétique, lorsque la dissolution a progressivement fait passer la concentration moyenne des premières couches en dessous d'une valeur critique $\left(c_{\text {moy }}=0,18\right)$, le système bascule vers un nouvel état d'équilibre local où le précipité se trouve sur les couches 2 et 3 . Cet état est très similaire à celui du stade $\mathrm{A} 2$ obtenu lors de la dissolution d'une MC (figure 1).

L'équilibre local ainsi formulé permet également de comprendre pourquoi l'étape B2 (une seule couche flottante de cuivre) n'apparaît pas lors de la dissolution d'une seule monocouche de fer. Elle correspond à un équilibre local, dont la stabilité exige une concentration moyenne sur les cinq premières couches supérieure à 0,18 , et donc une quantité de fer supérieure à 5 x $0,18=0,90 \mathrm{MC}$.

Signalons enfin que pour une concentration moyenne et une condition de raccord données, il existe plusieurs états d'équilibre pour la couche mince de cinq plans. Ces différents états correspondent à 
différentes épaisseurs de la couche flottante, ou en d'autres mots, à un précipité de fer s'étant enfoncé plus ou moins loin de la surface. Ainsi, dans le cas de la figure 3 , le premier équilibre local exploré par la cinétique (où le précipité de fer est situé en $p=1$ et 2) coexiste avec le second (précipité en $p=2$ et 3 ) et est même d'énergie libre légèrement moins favorable. Pourtant, lors du chemin cinétique, il est parcouru entièrement jusqu'à sa disparition, le système basculant alors dans le second équilibre. Cette exploration du premier équilibre local est due essentiellement à des raisons cinétiques: le précipité, initialement sur les plans 0 et 1, doit passer par l'état intermédiaire sur les plans 1 et 2 (équilibre 1 ou étape B2) avant d'atteindre les plans 2 et 3 (équilibre 2 ou étape B3).

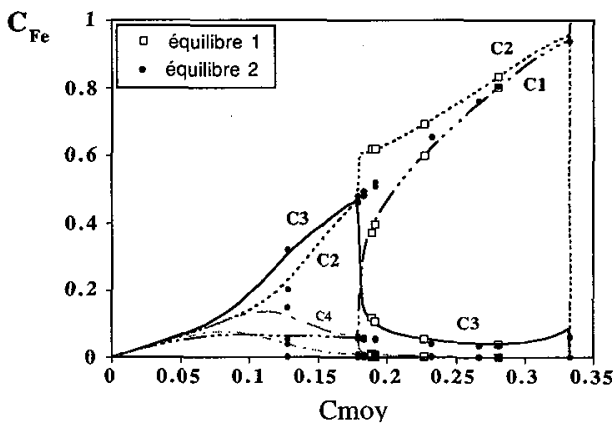

Figure 3: Mise en évidence de l'équilibre local. Les profils de concentration cinétiques et ceux d'équilibre obtenus pour une couche mince de cinq plans sont portés en fonction de la concentration moyenne sur ces cinq plans.

\section{CONCLUSION}

Pour un dépôt de fer sur un substrat de cuivre, nous avons montré, à l'aide du modèle KTBIM, l'apparition de couche(s) "flottante(s)" de cuivre à la surface, en accord avec les observations expérimentales. Dans le cas d'un dépôt de deux couches de fer, nous avons observé deux états successifs correspondant à une monocouche flottante de cuivre, puis à une bicouche, la cinétique de transition entre ces deux états étant rapide.

Ces différents états observês durant la cinétique peuvent perdurer sur des temps très longs. Ils correspondent en fait à des configurations en équilibre local, les transitions observées durant la cinétique correspondant à des transitions d'un état d'équilibre local vers un autre. Le paramètre contrôlant la transition est la concentration moyenne de la région en équilibre local, qui varie à cause de la dissolution progressive du dépôt.

L'extension spatio-temporelle du domaine de validité de l'équilibre local est en cours d'étude à la fois dans la structure C.C (présent travail) et dans la structure C.F.C. Concernant ce dernier point, remarquons que des phénomènes similaires (remontée des atomes de substrat, existence d'un ou plusieurs états métastables de longue durée avant le régime de dissolution fickien) ont été également observés et modélisés [12] dans la structure C.F.C (dépôt de Ni/Ag (100)). Ainsi le changement de structure cristallographique n'a eu que peu d'influence sur l'effet surfactant et le mode de dissolution ultérieur, tant qu'il ne remet pas en cause les deux forces motrices principales, à savoir la différence des énergies de surface et la forte tendance à la démixtion.

\section{Références}

[1] Steigerwald D.A., Jacob I., Egelhoff W.F., Surf. Sci. 202 (1988) 472-492

Detzel Th., Memmel N., Phys. Rev. B 49 (1994) 5599

[2] Senhaji A., Tréglia G., Legrand B., Barrett N.T., Guillot C., Villette B., Surf. Sci. 274 (1992) 297

[3] Legrand B., Saúl A., Tréglia G., Materials Science Forum 155-156 (1994) 165-188

[4] B.Aufray, H.Giordano, B.Legrand, G.Tréglia, Surf. Sci.307-309 (1994) 531-537

[5] Aldén M., Skriver H.L., Mirbt S., Johansson B., Surf. Sci. 315 (1994) 157-172

[6] Saúl A., Legrand B., Tréglia G., Phys. Rev. B 50 (1994) 1912-1921

[7] Salje G., Feller-Kniepmeier M., J. Appl. Phys. 48 (1977) xxx

[8] Ducastelle F., Order and Phase Stability in Alloys (North-Holland, Amsterdam, 1991)

[9] Tomanek D., Aligia A.A., Balseiro C.A., Phys. Rev. B 32 (1985) 5051

Tréglia G., Legrand B., Phys. Rev. B 35 (1987) 3226

[10] Laguës M., Domange J.L., Surf. Sci. 47 (1975) 77

[11] Martin G., Phys. Rev. B 41 (1990) 2279

[12] T. Bigault, rapport de stage (1994) 